\title{
A TRAFFIC MANAGEMENT SYSTEM APPROACH WITH THE IMPLEMENTATION OF ARTIFICIAL INTELLIGENCE ALGORITHMS
}

\author{
Shormee Saha \\ Lecturer, Department of Computer Science and Engineering, \\ Royal University of Dhaka, Banani, Dhaka, Bangladesh
}

\begin{abstract}
The traffic situation is one of the biggest problems in South Asian countries. Due to the increased rate of population and impact of industrialization, urbanization people's GDP has also increased resulting in abundant of vehicles. Traffic management systems have traditionally been limited to addressing the management of street signal lights. Algorithmic solutions to the current drawbacks have proved to be terribly restrictive, whereas expert system solutions have solely shown valid results with small signal networks. None of those approaches has addressed the requirement for management of the overall transportation system including the surface streets, interstate highways, public transportations, and emergency vehicle response etc. factors. Therefore it is necessary to design a distributed smart system with the implementation of advanced algorithms of artificial intelligence which will be capable of addressing the problems in traffic monitoring and controlling, congestion prediction, incident management and adaptive communication to the problem domain and will give the feasible solution of all these. The purpose of this paper is to provide an overview of the AI techniques that are conceivable to implement in order to handle the transportation issues in traffic safety, public transportations, traffic management, urban mobility etc. The paper also includes the challenges and limitations of $\mathrm{AI}$ algorithms in the same problem domain.
\end{abstract}

Keywords: Transportation system, Traffic Congestion system, Simulation, Artificial Intelligence, Modeling, Learning, Traffic Management.

\section{INTRODUCTION}

Intelligent technologies that are upgrading various sectors of our life with ease and comfort don't ignore the transportation system. For instance, automated transport systems and intelligent transport systems which collect information from the transportation behaviours and use data communication implemented to vehicles or their infrastructure. The primary objective of an advanced traffic management system is to manage the existing transportation resources efficiently in response to the dynamic traffic conditions. It must integrate each and every mode of transportation to provide an efficacious management solution. The solution. The main advantage is that the domain and problem descriptions are defined in a declarative language. That is why, it is easier for the traffic engineers to introduce new actions, sensor information, data and metrics. The model should be able to get updated automatically by using the AI learning techniques. The proposed system can be considered as an instance of a completely autonomous system which incorporates the properties like selfmonitoring, self-diagnosis, self-optimization, selfhealing, self-healing and self-adaptation.

\section{ARCHITECTURE}

A planning monitoring architecture can be used to provide a framework to integrate different components of our system. Figure 1 shows the sketch of the architecture designed. It starts from the EXECUTION module which receives the problem domain. Then the current state of the world, state, is captured and the problem initial state is set. The initial goal set also can be set by the GOAL AND METRIC GENERATION module. The PLANNING module is called by the MONITORING module to obtain a plan and the 


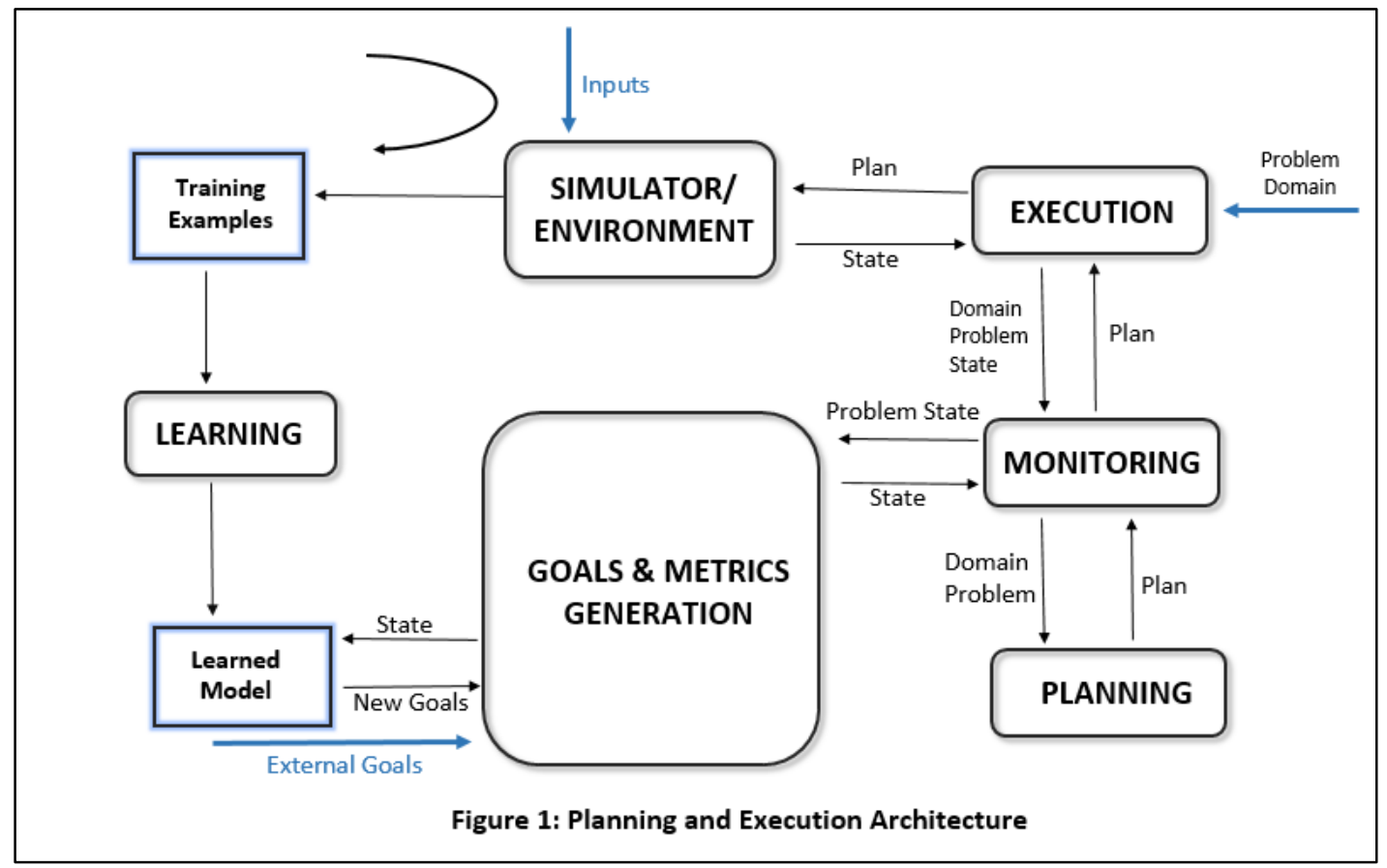

actions of the plan are sent back to the EXECUTION module. After the actions being executed, the MONITORING module receives the required knowledge (current state, problem domain etc.) from the EXECUTION module so that a new planningexecution-monitoring cycle can be initiated. If the execution didn't generate the expected changes (reduction in traffic density in some streets), it will produce a new goal and a new initial state for a new call to the planner. The GOAL\&METRIC GENERATION module combines those goals with possible external ones (given directly by the traffic controllers) to update the problem. A Simulator can substitute the environment in some domains.

One of the biggest challenges in this proposed architecture is the generation of new goals. Here, Machine learning techniques will be implemented to hypothesize when new goals should be generated to anticipate future problematic streets. In a training step, the traffic behaviour during some time intervals, under discrete traffic conditions is observed to generate examples. Then a learning algorithm will utilize those examples to construct a model, such that for any new state it must return a new goal. The learning process is assumed to be online, prior to the actual use of the APbased-system, but it could be done offline as well.

\section{PLANNING TASKS}

According to the design the problem domain and the simulator would take input from the environment which may include traffic management decisions, traffic congestion reports, model predictions, police/public safety reports, transit schedules and status, event schedules and attendance, airport schedules and status, commercial trucking, ship line schedules and status, commercial traffic reports, weather reports, incident reports, emergency calls, traffic spotters, video cameras, traffic sensors, cellular phones, sites of interest, directory of accommodations, directory of services etc. This information provides an extensive view of the current status of the transportation environment and constructs the core database which the system would draw upon during the decision-making process. After the successful implementation of decision-making steps, the system would directly supply the users with information on

- The best mode of transportation available

- The best route suggestion

- The existence of services and whether they are obtainable or not

- Parking availability

- In-vehicle hazard warnings and road signing

- Predicted congestion area with real-time analysis 


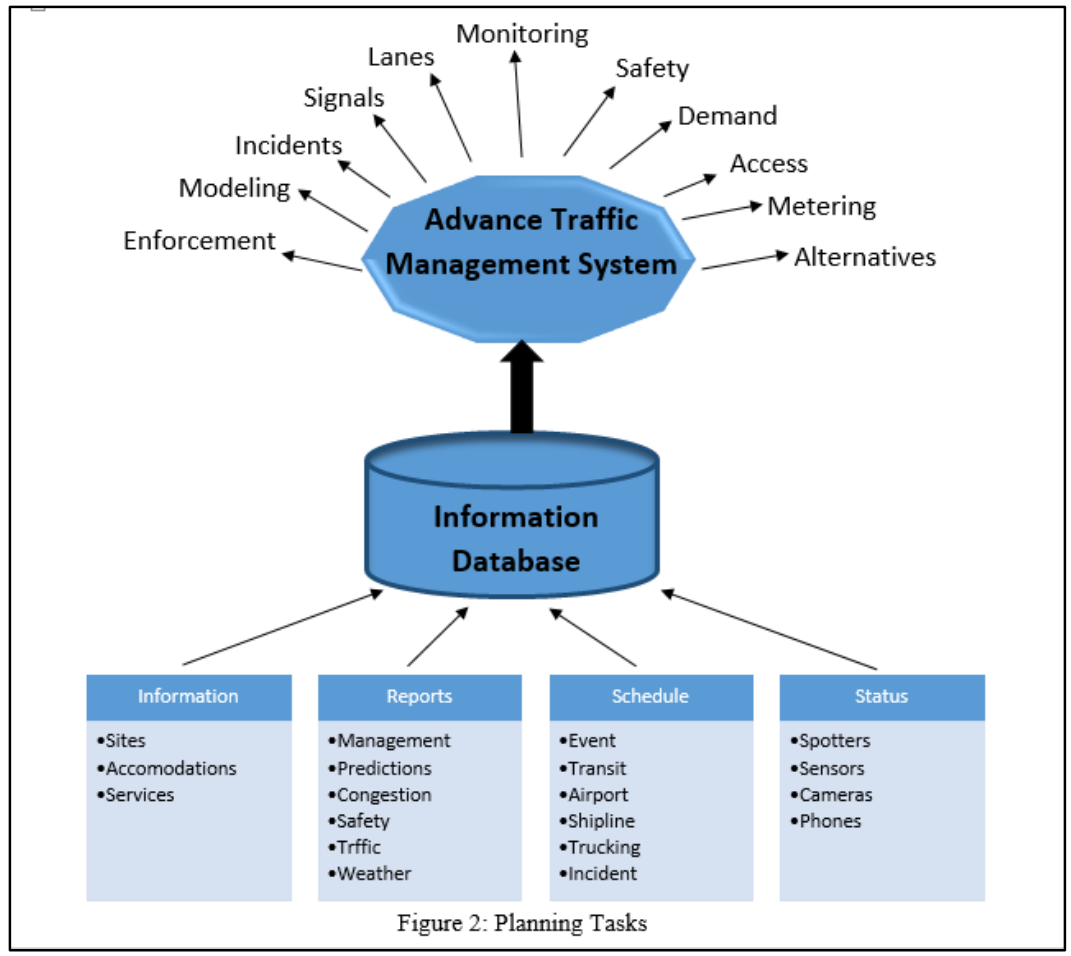

Utilizing the information database as described earlier, the system will be able to manage traffic by making control decisions on all aspects of traffic flow and implementing them. Decisions will optimize the traffic flow and will be able to address environmental impacts. Specific tasks of the system will include:

- Traffic congestion monitoring

- Predictive traffic flow modelling

- Coordinated signalization of surface street lights

- Opening of reserved lanes and reversible lanes

- Ramp metering onto congested highways

- Electronic enforcement of traffic regulations

- Incident detection algorithms

- Request for police or emergency vehicles

- Demand management

- Automated toll collection

\section{LEARNING TRAFFIC BEHAVIOR}

Whenever a new goal arrives, the task of learning is mandatory to predict the density level of the streets so that their congestion can be anticipated and appropriate goals can be generated for the planner. In this section, the learning task will be defined. We articulate this problem as a time series prediction one, using Statistical Relational Learning.

Statistical relational learning (SRL) is a sub-discipline of artificial intelligence and machine learning that is concerned with domain models that exhibit both uncertainty (which can be dealt with using statistical methods) and complex, relational structure. In this case, it is conjectured that the structured layout of a city can have influence over the density levels of some interconnected streets. SRL suits the first-order logical representations like the one used in PDDL.

\section{REPRESENTATION}

The representation is based on a subset of the predicates that are used in the planning of traffic domain.

We modify some of those predicates, to represent the time steps, adding corresponding time steps. We recognize two types of predicates: the static and the dynamic one. The static part of a city is represented by the connection predicate. All the connection predicates constitute the entire city network.

The dynamic part of the city is formed by the density of the streets and the state of the traffic lights. 


\section{International Journal of Engineering Applied Sciences and Technology, 2019 \\ Vol. 4, Issue 6, ISSN No. 2455-2143, Pages 1-6 \\ Published Online October 2019 in IJEAST (http://www.ijeast.com)}

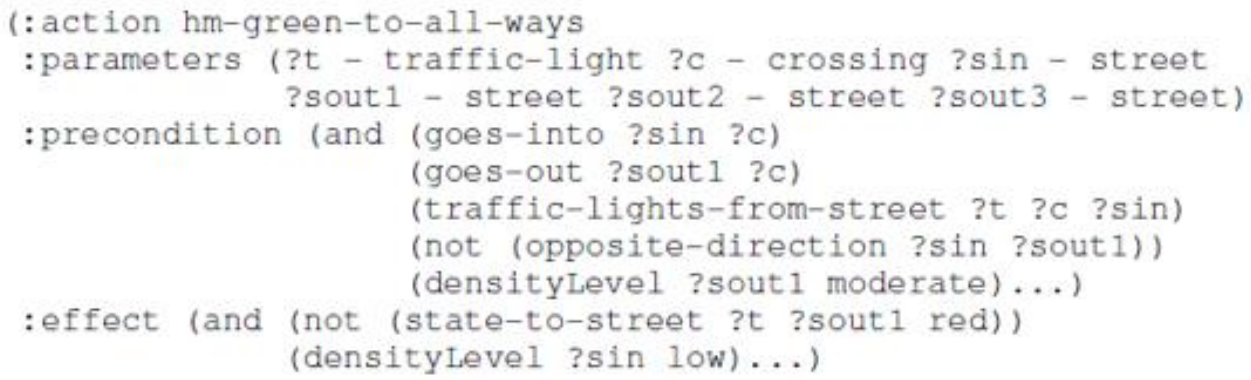

Figure 3: Part of an example description of a PDDL action.

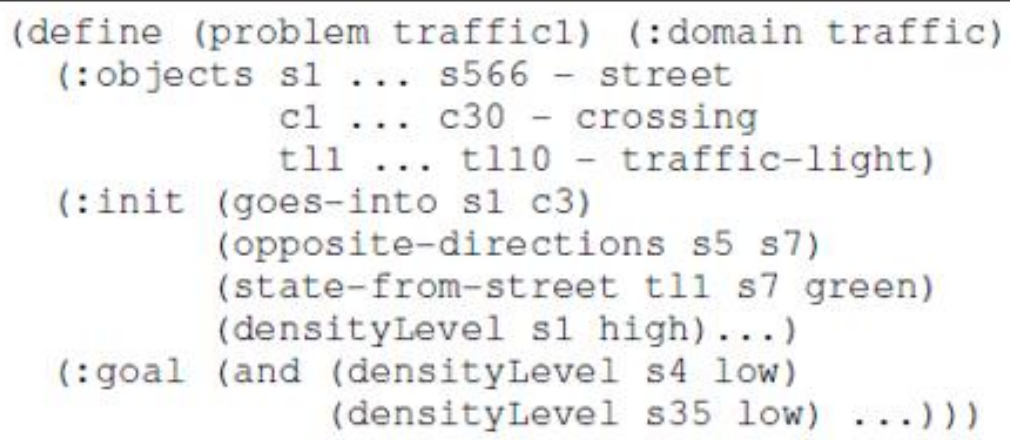

Figure 4: Part of an example PDDL problem file.

\begin{tabular}{c|c} 
Predicate & Type \\
\hline density(st,l) & Dynamic \\
connection(st,st) & Static \\
open $X(\mathrm{tl}, \mathrm{st})$ & Dynamic \\
density $L X(\mathrm{st})$ & Dynamic
\end{tabular}

Table 1: Predicates used in the learning task. X represents the time step. L represents the density level.

A green traffic light tl at time step X, located at street st is represented by the openX(tl, st) predicate. The density LX(st) predicate represents that at time step X a street st has a density level L. L can take the values like very low, low, moderate, high, very high. The last predicate density (st,l), represents the current density level 1 of the street st. This will indicate the class of each of the examples.

\section{ALGORITHMS}

An algorithm has been developed at the Katholieke Universiteit Leuven, that learns a predicate logic theory by means of decision trees. Logical decision trees are a first-order logic upgrade of the classical decision trees used by propositional learners. The resulting trees can directly be used for classification of unseen examples, but they can also easily be transformed into a logic or Prolog program. Following that algorithm, an example is shown below which can be implemented in our desired system. Here, A represents the example id and the other letters are used as the predicate's arguments.

A minus symbol before the variable indicates that it is new in the tree, whereas a variable symbol appearing alone means that is has been referenced before. The classes that are required to be predicted appear in the leaf nodes of the tree between brackets.

\section{RESULTS ON TRAFFIC MANAGEMENT}

The main purpose is to test whether a traffic control system would improve its performance using some predictive model of the traffic. We need to work on several simulation scenarios based on three parameters, which are the size of the network (medium and large), the fluency of traffic (fluent or congested) and the evaluated time period (an hour to a day).

While using the learning model, it should predict the density at each street at each time step, using the $\mathrm{X}$ time step as input. Detecting a high density at any subset of the street sections, it generates goals to lower 


\section{International Journal of Engineering Applied Sciences and Technology, 2019 \\ Vol. 4, Issue 6, ISSN No. 2455-2143, Pages 1-6 \\ Published Online October 2019 in IJEAST (http://www.ijeast.com)}

the density of those sections. These new goals together and the current traffic state create a PDDL planning problem which is given as input to the planner. The system must check for new goals every fifty seconds using the prediction model built with the five minute time steps.

We compare our system with a static or default one and also with a Reactive system, which acts locally on each traffic signal light and keeps a longer phase for the green light on those streets where density is currently high.

\section{CONCLUSION}

In this paper, we have suggested a dynamic approach to automated planning and relational learning. We have seen that, by adding a learning component to a planning system, which can predict the city-state, we can highly increase its autonomy. It can automatically produce its own goal and assist the planner to initiate the planning process sooner. The system can be tested in several traffic control scenarios to demonstrate its ability to anticipate goals can lead to a better control performance than using only static traffic light signals. By knowing the street density our proposed system must be capable of modelling a wide variety of circumstances that affect the traffic behaviour. The circumstances might be like adverse weather condition or different days or hours, road blocking or big accidents etc. This approach is expected to alleviate congestions caused by them by suggesting efficient planning. In future work, some advanced features likeability to learn how to anticipate goals with externally supplied goals, reactively generated ones or internally supplied ones etc. can be integrated. The real-time applicability of the system can fully be acknowledged by implementing it in irregular city networks such as Mumbai, Delhi, Dhaka, Bogota, Moscow, Bangkok etc.

\section{REFERENCES}

1. Rndr. Šusteková D. Phd., rndr. Knutelská M. Phd. (2015). How is the artificial intelligence used in applications for Traffic management, Scientific Proceedings Xxiii International ScientificTechnical Conference "Trans \& Motauto '15", (page 91-94). Faculty of Operation and Economics of Transport and Communications, Department of Quantitative Methods and Economic Informatics, University of Žilina, Žilina, Slovak Republic.

2. Khaled Abdul Rahman Jomaa , (2016). An Artificial Intelligence Techniques and Simulation Model to Control a Traffic Jam System in Malaysia (Review Paper), Asian Journal of Business and
Management (ISSN: 2321 - 2802), Faculty of Industrial Management, University Malaysia Pahang, 26300, Pahang, Malaysia.

3. Erik björck Fredrik omstedt (2018), degree project in technology, A comparison of algorithms Used in traffic control Systems, kth royal institute of technology.

4. Malte Helmert, (2016), An Introduction to PDDL, AI Planning.

5. Rusul Abduljabbar, Hussein Dia, Sohani Liyanage and Saeed Asadi Bagloee (2019). Applications of Artificial Intelligence in Transport: An Overview, in www.mdpi.com/journal/sustainability Department of Civil and Construction Engineering; Swinburne University of Technology, Hawthorn.

6. John F. Gilmore and Khalid J. Elibiary (2015), AI In advanced traffic management systems in AAAI Technical Report WS-93-04 , (Page 57- 65). Artificial Intelligence Group, Computer Science and Information Technology Lab, Georgia Tech Research Institute, Georgia Institute of Technology, Atlanta, Georgia.

7. Blockeel and De Raedt, (1998) Hendrik Blockeel and Luc De Raedt. Top-down induction of firstorder logical decision trees. Artificial intelligence, 101(1):285-297, 1998.

8. Bonet and Geffner, (2005). Blai Bonet and H'ector Geffner. mGPT: A probabilistic planner based on heuristic search. JAIR, 24:933-944, 122005.

9. Box and Waterson, (2012). Simon Box and Ben Waterson. An automated signalized junction controller that learns strategies from a human expert. Engineering applications of artificial intelligence, 25(1):107-118, 2012.

10. Gokulan and Srinivasan, (2010). Balaji Parasumanna Gokulan and Dipti Srinivasan. Distributed geometric fuzzy multiagent urban traffic signal control. Intelligent Transportation Systems, IEEE Transactions on, 11(3):714-727, 2010.

11. Guli`c et al., (2015) Matija Guli`c, Ricardo Olivares, and Daniel Borrajo. Using automated planning for traffic signals control. In Working Notes of ARTS-COST 2nd competition, 2015.

12. Guzm'an et al., (2012) C'esar Guzm'an, Vidal Alc'azar, David Prior, Eva Onaind'ia, Daniel Borrajo, Juan Fdez-Olivares, and Ezequiel Quintero. PELEA: a domain-independent architecture for planning, execution and learning. In Proceedings of ICAPS'12 Scheduling and Planning Applications woRKshop (SPARK), pages 38-45, Atibaia (Brazil), 2012. AAAI Press. 
13. Hamilton et al., (2013) Andrew Hamilton, Ben Waterson, Tom Cherrett, Andrew Robinson, and Ian Snell. The evolution of urban traffic control: changing policy and technology. Transportation planning and technology, 36(1):24-43, 2013.

14. Kuyer et al., (2008) Lior Kuyer, Shimon Whiteson, Bram Bakker, and Nikos Vlassis. Multiagent reinforcement learning for urban traffic control using coordination graphs. In Machine learning and knowledge discovery in databases, pages 656671. Springer, 2008. 